\title{
Metodologia Adotada na Reconstrução 3D da Estação Saudade, em Ponta Grossa - PR
}

\author{
Matheus Fratus*, Mauren L. S. Coelho de Andrade ${ }^{\S}$ \\ *Universidade Tecnológica Federal do Paraná, Ponta Grossa, Paraná, Brasil \\ Email: fratus@alunos.utfpr.edu.br \\ §Universidade Tecnológica Federal do Paraná, Ponta Grossa, Paraná, Brasil \\ Email: mlsguario@utfpr.edu.br
}

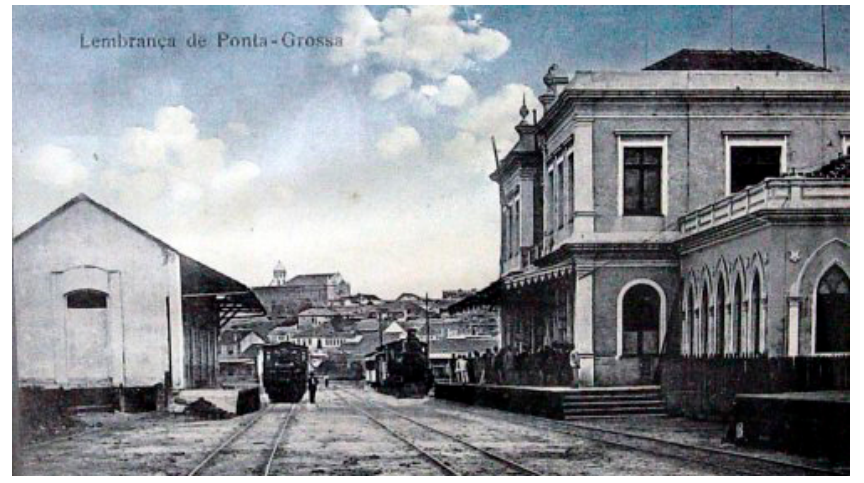

Figura 1. Cartão postal de Ponta Grossa. (aproximadamente 1909)

Abstract-This paper presents the methodology adopted in the digital reconstruction of the São Paulo - Rio Grande railway station (Saudade Station), to produce a real time virtual visualization application in the future. The station is a cultural heritage site of the Brazilian state of Paraná and one of the oldest buildings in the city of Ponta Grossa. The methodology presented here is based primarily on the use of photographs as a reference for $3 D$ modeling, seeking visually realistic results and using only free tools such as Blender and Cycles.

Resumo-Este artigo apresenta a metodologia adotada na reconstrução digital da estação ferroviária São Paulo - Rio Grande (Estação Saudade) com o objetivo de posteriormente gerar uma aplicação de visualização virtual em tempo real. A estação é patrimônio cultural do Paraná e uma das construções mais antigas da cidade de Ponta Grossa. A metodologia apresentada é baseada principalmente no uso de fotografias como referência para modelagem 3D, utilizando somente ferramentas gratuitas como o Blender e buscando resultados visualmente realistas.

\section{INTRODUÇÃO}

Repleta de história e monumentos arquitetônicos de época, Ponta Grossa, cidade no leste do Estado do Paraná, completa, em 2023, duzentos anos de fundação. A Estação São Paulo Rio Grande (Figura 1), conhecida hoje por Estação Saudade, foi a segunda estação ferroviária da cidade. Construída ainda em 1900, ela serviu como alternativa ao grande fluxo de passageiros e mercadorias que não era mais suportado pela pequena Estação Paraná. [1]

$\mathrm{Na}$ época foi considerada uma estação de primeira classe, destacada pelo seu porte, localização e características arqui-

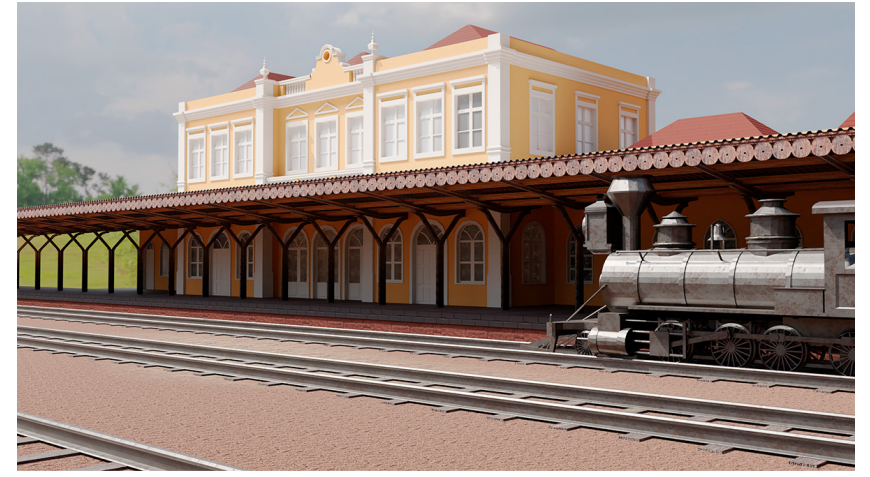

Figura 2. Reconstrução 3D feita com o Blender [2].

tetônicas de vertentes neoclássicas e art-nouveau. Além de servir como posto de entre cargas também era passagem do trem internacional Itararé-Uruguai.

A estação foi ampliada em 1908 e em 1990 foi tombada como Patrimônio Cultural do Paraná. Atualmente o edifício se encontra fechado para restauração e suas características originais ainda estão preservadas.

A reconstrução digital de patrimônios históricos é tema de trabalhos no mundo todo há vários anos e diversas técnicas são adotadas para esse fim [3] como escaneamento 3D a laser para a geração de uma nuvem de pontos representando a superfície do objeto a ser retratado (a exemplo o trabalho de Andrew Tallon [4] utilizado na reconstrução da catedral de Notre-Dame de Paris após o incêndio de 2019), utilização de drones [5] também para escaneamento de pontos $3 \mathrm{D}$, fotogrametria, que é a utilização de referências fotográficas para medições como base para modelagem 3D, ou ainda abordagens que misturam mais de uma das técnicas anteriores [6].

Desenvolver um modelo a partir de fotografias, como proposto neste artigo, permite que o projeto possa ser facilmente realizado sem a necessidade de equipamentos e algoritmos especiais para captação de pontos e recriação de malha. Também é interessante quando o acesso ao local é restrito ou não existe mais. Ainda, apesar de não tão preciso como um escaneamento 3D a laser ou utilizando drones, um modelo com malha mais simples e com extensões de arquivo comuns, permite que este seja exportado para fins diversos sem muita 
dificuldade, como aplicações em tempo real e animações.

Resultado de uma série de trabalhos apresentados por um grupo de alunos de Ciência da Computação, este artigo descreve a metodologia adotada na reconstrução digital através da modelagem 3D deste importante patrimônio histórico. A técnica aqui apresentada pode ser facilmente aplicada a outras reconstruções digitais onde os edifícios em estudo não estejam fisicamente disponíveis por limitação de acesso ou mesmo por terem sido destruídos por desastres naturais, incêndios ou guerras. Ainda, a metodologia não depende de recursos caros como scanners laser ou drones e pode ser implementada sem muito esforços e com resultados visualmente satisfatórios, sendo necessário no mínimo um computador pessoal intermediário para a execução dos softwares descritos e acesso à fontes e fotografias históricas .

\section{Materiais e Métodos}

Para o recolhimento de dados históricos foi formada uma pequena equipe de pesquisa composta pelos alunos da disciplina de Computação Gráfica. Durante uma semana a equipe visitou os monumentos a serem retratados, levantou informações em lojas de fotografia, visitou museus e entrou em contato com pessoas que possuem acervos pessoais de fotografias antigas da cidade. No caso, a principal fonte de dados foi a Casa da Memória Paraná, em Ponta Grossa. Estabelecimento mantido pela prefeitura e que possui o maior acervo físico de documentos históricos sobre o município.

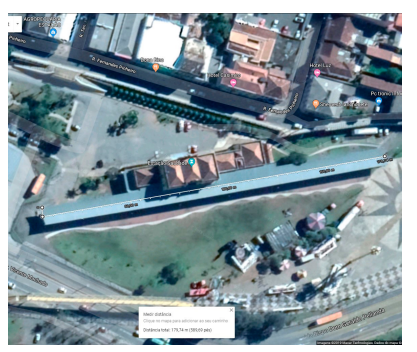

(a) Visão superior e ferramenta de medida.

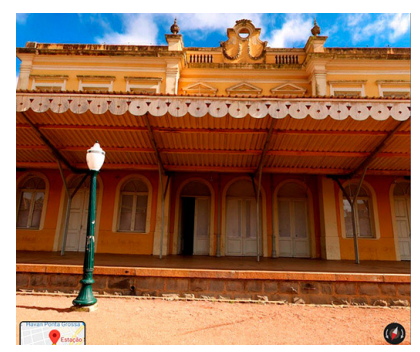

(b) Visão $360^{\circ}$ frontal do prédio.
Figura 3. Ferramentas do Google Maps.

Outra importante fonte de dados foi o Google Maps que oferece tanto uma visão em $360^{\circ}$ da parte frontal do edifício (Figura 3b) quanto uma ferramenta de medida em imagens de satélite (Figura 3a).

Para a criação dos modelos e das visualizações foi optado pela ferramenta Blender [2] (Figura 4), software de manipulação 3D open source mantido e desenvolvido pela Blender Fundation. A escolha se alinha com a praticidade de se utilizar um software que é ao mesmo tempo gratuito, utilizado na indústria e oferece todas as ferramentas necessárias para manipulação 3D, como modelagem, texturização, criação de materiais e renderização.

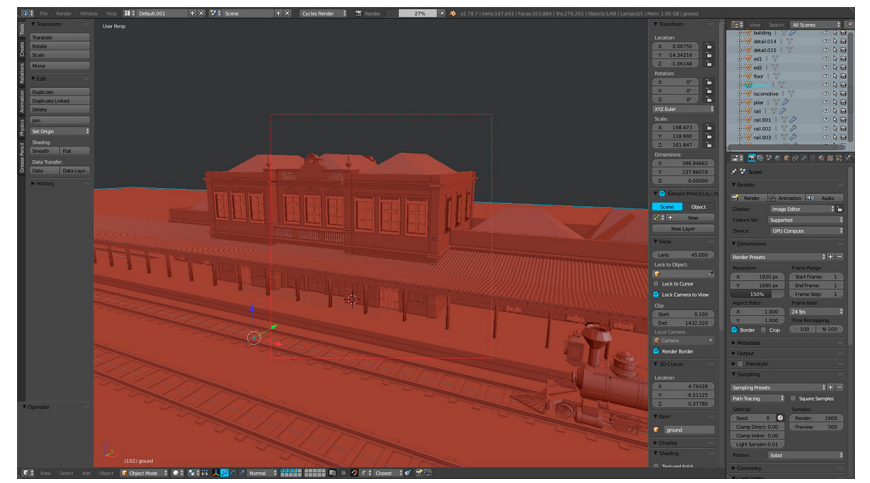

Figura 4. Interface do Blender [2] e modelo 3D da estação.

\section{RESUltados}

\section{A. Modelagem}

Cruzando as informações de várias fotografias antigas e recentes com as imagens de satélite foi possível realizar uma modelagem com uma boa exatidão de medidas e detalhes (figura 4).

Com essa abordagem de modelagem manual o tempo para criação dos objetos acaba tendo relação direta com a experiência do modelador e sua familiaridade com a ferramenta adotada. Mesmo assim o processo de aprendizagem da ferramenta para usuários novos é rápido e a assimilação de conceitos simples como manutenção da malha 3D é suficiente para a criação de modelos consistentes.

Uma outra abordagem de modelagem que poderia ser adotada é a procedural (não destrutiva). Hoje esse tipo de modelagem, utilizada em softwares como o Houdini [7], é cada vez mais utilizada pela praticidade de se realizar modificações e variações em modelos sem que seja necessário retrabalhos exaustivos. No caso em estudo, reconstrução de monumentos, esta abordagem não foi escolhida, pois, desde que o modelo seja feito com o devido cuidado, não são necessárias modificações drásticas posteriores nem variações para se criar objetos distintos. O objetivo é um modelo único e preciso.

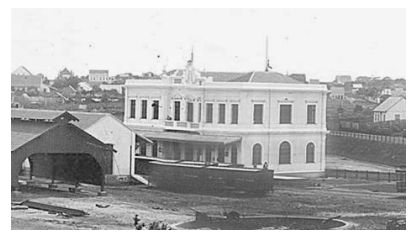

(a) Estação em sua primeira configuração. Aproximadamente 1900.

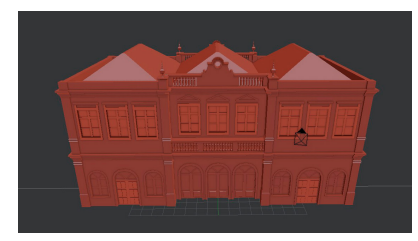
do prédio. (b) Modelo 3D da primeira versão

\section{Figura 5. Primeira versão do prédio.}

Houveram diversas expansões durante a história da estação, como a adição de prédios anexados e uma nova área de embarque. Assim durante a modelagem foi procurado preservar essas mudanças graduais para que o monumento pudesse ser representado em seus vários anos diferentes (figura 5b).

Ainda sobre a modelagem, foi optado por representar uma boa quantidade de detalhes enquanto se preservava uma baixa 
quantidade de polígonos, permitindo futuramente produzir um aplicativo que reproduza o modelo em tempo real com baixas exigências de hardware.

\section{B. Texturização e Criação de Materiais}

Como o resultado visual buscado consiste no realismo a criação de materiais, texturização e renderização foi baseada em uma abordagem física. Para tal o sistema de desenvolvimento de materiais do renderizador Cycles [8] foi escolhido. O Cycles é um renderizador path tracer baseado em física é que é nativamente integrado ao Blender. O processo de renderização é detalhado no subtópico "Renderização".

A criação de materiais no Cycles é feita através de um sistema procedural não destrutivo baseado em nodes (nós). Há diversos tipos de nó para controle de diferentes aspectos do material, como fontes de textura, fontes de mapeamento uv (utilizado na associação de um ponto da superfície de um objeto e pixels em uma imagem 2D), modificadores e, principalmente, os shaders, responsáveis pela caracterização física do material.

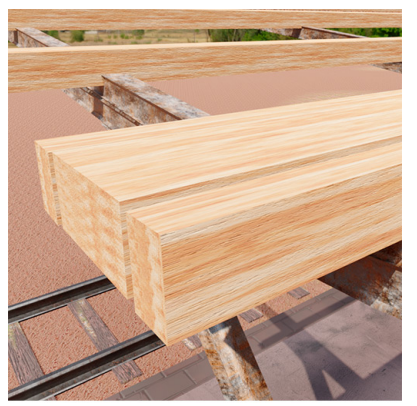

(a) Detalhe do material representando madeira.

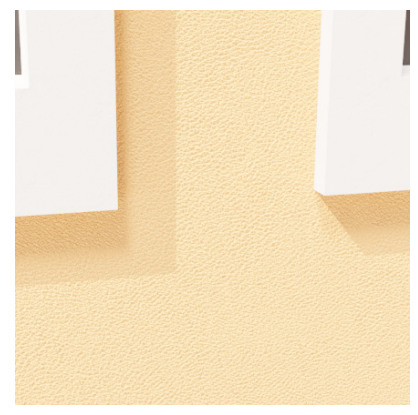

(b) Detalhe da textura da parede.
Figura 6. Exemplos de materiais e texturas utilizados no projeto.

No projeto em questão todos os materiais foram criados através de uma abordagem de renderização baseada em física (PBR, na sigla em inglês). Para tal o principal nó utilizado foi o shader Principled BSDF, que se baseia no modelo de shader PBR desenvolvido pela Disney [9]. Este nó possui como principal característica ser tão fisicamente fiel quanto fácil de modificar e utilizar. Ele reúne diversas propriedades físicas dos materiais como índice de refração, "aspereza" (roughness) e comportamento condutor ou dielétrico, bem como ajusta automaticamente efeitos baseado nos outros parâmetros, como o fresnel. Essas propriedades são suficientes para reproduzir a maioria dos objetos reais, e utilizando texturas previamente pensadas para PBR é possível atingir rapidamente resultados satisfatórios. No geral esse tipo de textura é desenvolvida de forma procedural e disponibiliza mapas de intensidade específicos para vários dos parâmetros do Principled $B S D F$, o que permite gerar padrões de textura infinitos e alterar propriedades como índice de reflexão e vetores normais em pontos específicos da superfície do objeto.

\section{Iluminação}

A iluminação da cena foi mapeada a partir de uma imagem panorâmica HDR (do inglês High Dynamic Range) que cobre todos os $360^{\circ}$ de visualização. Este método de iluminação utiliza as informações de intensidade luminosa e cor proveniente dos pixels da imagem panorâmica como fontes de luz da cena, representando fielmente as características de um ambiente específico.

$\mathrm{O}$ que difere uma imagem HDR de uma imagem comum é a quantidade de dados que cada uma consegue guardar sobre a intensidade luminosa. Enquanto as imagens com 8 bits por canal de cor conseguem discretizar apenas 256 intensidades diferentes, as imagens HDR possuem até 32 bits por canal, podendo representar uma quantidade de informação absurdamente maior. Isso possibilita que uma fonte luminosa como o Sol possa ser representada com sua intensidade real em relação à quantidade de luz refletida pelos objetos na superfície da terra, por exemplo, e sem perda de detalhes.

A criação desse tipo de imagem envolve equipamento específico, porém, hoje, bibliotecas virtuais oferecem diversas opções de imagens HDR em ambientes variados, o que em muitos dos casos é suficiente para representar um ambiente modelado em computador.

No Blender esse tipo de iluminação é facilmente configurado através do material padrão responsável pelo ambiente (background) da cena. Basta criar um nó de Environment Texture com o caminho para a imagem HDR e associá-lo ao nó Background já existente.

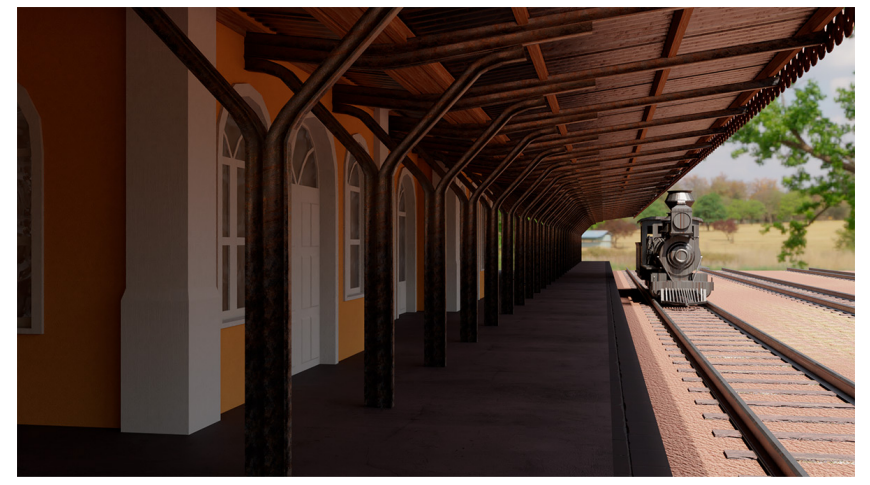

Figura 7. Renderização utilizando o Cycles [8], renderizador baseado em física e integrado ao Blender. A imagem de fundo não pretende retratar o ambiente original, trata-se de uma imagem utilizada como mapa de iluminação.

\section{Renderização}

A renderização do projeto foi feita com o Cycles [8], renderizador baseado em física já incluído no Blender. A escolha desse renderizador se alinha com a necessidade de um resultado que simula a realidade já que ele se baseia no traçado de partículas de luz e suas interações com materiais.

De forma simplificada o traçado de raios utilizados pelos renderizadores baseado em física consiste na simulação do caminho da luz emitida por fontes de iluminação e sua interação com os objetos 3D, assim como no mundo real. 
Os algoritmos de ray tracing são responsáveis por simular esses caminhos. Geralmente a simulação ocorre no sentido inverso, partindo da câmera, já que os únicos raios relevantes para gerar uma imagem são aqueles que eventualmente chegam no observador. Existem diferenças entre métodos como o ray tracing clássico, path tracing e bidirectional path tracing que não serão abordados aqui, mas uma descrição razoável de seus funcionamentos é que esses algoritmos verificam se um raio partindo de um pixel colide com algum objeto e a partir das características físicas descritas pelo material e pela superfície associado a ele, predizem a origem desse raio, que pode ser uma fonte de luz, outro objeto (resultado de uma reflexão), ou nenhuma (que é o mesmo que dizer que não existe luz associada a esse raio). Combinando as informações adquiridas pelo raio no seu caminho é produzido a cor final do pixel.

A vantagem mais evidente do ray tracing frente outros métodos como a rasterização é o resultado próximo da realidade. Enquanto o traçado de raios se baseia na simulação física da luz, a rasterização, geralmente utilizada em aplicações de tempo real, projeta os vértices das primitivas geométricas em um plano 2D, que representa a imagem, e a partir daí as formas geométricas com suas características são descritas através de algoritmos que simulam interações do objeto com a luz (shaders) através de artifícios como mapas de reflexão previamente gerados e operações envolvendo vetores normais.

As pré-visualizações renderizadas com o Cycles podem ser vistas nas figuras 2,7 e 6 .

\section{E. Baking}

Um dos objetivos futuros do projeto é criar uma aplicação que possa ser executada em tempo real com uma boa acurácia visual e que ainda assim seja praticável em hardwares mais modestos como computadores pessoais simples e smartphones.

Uma técnica comum em aplicações 3D de tempo real com visual realista é o baking. Um processo que consiste em armazenar a informação de iluminação calculada pela renderização diretamente nos objetos. A ideia por trás é simples: em vez de o produto da renderização ser armazenado na superfície de um plano de visualização que representa a imagem, o resultado da interação dos raios emitidos pelas luzes com os objetos é gravado em uma textura com coordenadas mapeadas na superfície dos objetos.

Outra possibilidade bastante discutida atualmente seria o ray tracing em tempo real. Mesmo que essa técnica gere um resultado fisicamente preciso e visualmente mais realista, hoje, para que esta seja praticável em tempo real necessita de hardware específico, até o momento oferecido pela arquitetura Turing da Nvidia em suas unidades de processamento gráfico (GPU, na sigla em inglês).

Umas das desvantagens de se utilizar a técnica de baking é que a iluminação da cena passa a ser estática. Como consequência efeitos dinâmicos deixam de ser diretamente simulados, como os reflexos, que mudam de acordo com a posição do observador, e interações entre objetos em tempo real, como sombras e cores.

\section{Conclusão e Trabalhos Futuros}

Foi apresentado neste artigo a metodologia adotada e o resultado da reconstrução digital da estação ferroviária São Paulo - Rio Grande (Estação Saudade) da cidade de Ponta Grossa.

Ainda há muito o que ser melhorado no projeto. Uma modelagem mais fiel dos detalhes e proporções da construção, bem como um trabalho extra na criação dos materiais e texturas, representando desgastes causados pela natureza e pela presença humana, podem trazer mais veracidade à simulação $3 \mathrm{D}$.

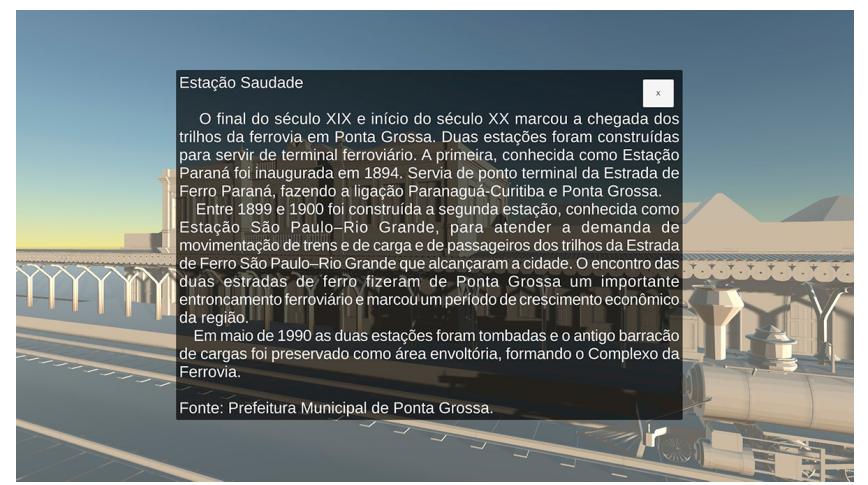

Figura 8. Aplicação de teste na engine multiplataforma Unity. Neste exemplo o usuário vê uma mensagem sobre a história do prédio e pode movimentar uma câmera virtual para observar a construção de diversos ângulos.

Como trabalho futuro está planejada a criação de uma simulação em tempo real do monumento utilizando a engine Unity [10] (Figura 8) e que será acessível através de navegadores. Nessa simulação, além de ser apresentado à história do monumento e suas mudanças ao decorrer das décadas, o internauta poderá caminhar digitalmente pela reconstrução 3D.

A UTFPR-PG realiza um projeto em comemoração ao aniversário de 200 anos da cidade de Ponta Grossa. Nele será reconstruído digitalmente os diversos pontos históricos do município. Além da relevância social frente a preservação do passado brasileiro, o projeto pretende criar um ambiente de pesquisa na área de computação gráfica junto aos estudantes de graduação, fomentando novas descobertas e técnicas.

\section{REFERÊNCIAS}

[1] (2019) Prefeitura de ponta grossa, estação são paulo - rio grande (conhecida como estação saudade). [Online]. Available: http://www. pontagrossa.pr.gov.br/estacao-saudade

[2] (2019) Blender foundation. [Online]. Available: https://www.blender.org

[3] D. D. Mascio, "3d digital reconstructions of lost buildings, a first critical framing," 2016.

[4] A. Tallon, "A la lumière du laser," in Le Nouvel Observateur, hors-série 80, 66-67. Le Nouvel Observateur, 2012.

[5] Soria-Medina, "Reconstrução 3d de monumentos históricos a partir de imagens obtidas com rpas," 2018.

[6] E. Dezen-Kempter, "Escaneamento 3d a laser, fotogrametria e modelagem da informação da construção para gestão e operação de edificações históricas," 2015

[7] (2019) Sidefx houdini. [Online]. Available: https://www.sidefx.com

[8] (2019) Cycles. [Online]. Available: https://www.cycles-renderer.org

[9] B. Burley, "Physically-based shading at disney," 2012.

[10] (2019) Unity technologies. [Online]. Available: https://unity.com 